\title{
Moderne Senologie aus Sicht der radiologischen Diagnostik
}

\section{Müller-Schimpfle}

Eine spannende Zeit - so könnte der Untertitel lauten für die folgenden Betrachtungen zur Entwicklung der modernen Senologie in den letzten 30Jahren aus Sicht der radiologischen Diagnostik. Die Radiologie hat in dieser Zeit mit der Entwicklung der Gerätetechnologie einen fantastischen Schub erhalten und zum Wohle ihrer Patienten nutzen können.

In den 60er- und 70er-Jahren wurden mit der Entwicklung moderner Mammografiegeräte, wie sie dann auch in den letzten 30 Jahren zum Einsatz kamen, die Screeningstudien erst möglich, die in den 70er- und 80er-Jahren die wesentliche Erkenntnis der Mortalitätsreduktion erbrachten. Lanyi nannte die 30Jahre zwischen 1951 und 1980 „die Geschichte einer allmählich verlorengegangenen Hoffnung, auf einfachste Weise Brustkrebserkrankungen diagnostizieren zu können“ [1]. Die nächsten 30Jahre zwischen 1981 und 2011 könnte man als die Geschichte einer Hoffnung auf Sterblichkeitsreduzierung durch Früherkennung bezeichnen. In den 80ern wurden die initialen großen skandinavischen Screeningstudien veröffentlicht, das britische und niederländische Screeningprogramm begonnen, in den 90ern wurde das Mammografiescreening in diesen Ländern für die nationale Gesundheitsfürsorge akzeptiert.

In Deutschland hat sich - bedingt durch unterschiedliche Gesundheitssysteme zu den Nachbarländern, aber auch unterschiedliche Einschätzungen - zunächst eine andere Entwicklung für die Mammografie-Reihenuntersuchung ergeben. Durch die Empfehlung eines selektiven Risikogruppen-Screenings der Kassenärztlichen Bundesvereinigung 1979 war die Mammografie in der Folge in Deutschland liberaler verfügbar als in den meisten anderen Ländern. Ein maßgeblich durch Frischbier und Höffgen (sowie Barth und von Fournier) vorangetriebene Studie zur „Mammographie in der Krebsfrüherkennung“ zeigte in den 90er-Jahren die Bedürfnisse und Notwendigkeiten zur Einführung eines Screening-Programmes auch in Deutschland [2]. Zur Einführung des flächendeckenden Mammografiescreening kam es dann in Deutschland in Folge eines Allparteienbeschlusses aus dem Jahr 2002 in den Jahren zwischen 2005 und Anfang 2009 zu einer koordinierten Einführung eines bundesweiten Mammografiescreenings in der vertragsärztlichen Versorgung. Die Entwicklung der Röntgentechnologie hin zu immer kleineren Strahlendosen und immer höherer technischer Qualität, zuletzt durch die bei Verabschiedung des Screeningprogramms sicherlich noch unvorhersehbar schnelle Einführung der digitalen Mammografie, wurde allerdings durch ähnlich bahnbrechende Entwicklungen insbesondere der Ultraschall- und MRT-Tech- nologie in den 80er- und 90er-Jahren des letzten Jahrhunderts begleitet. So hat sich der Ultraschall durch die Entwicklung der Ortsauflösung wie auch Herausarbeitung der relevanten diagnostischen Parameter seine Rolle als das ergänzende Verfahren zur Mammografie in den 90er-Jahren erobert. An der Spezifität des Verfahrens wird durch Beiträge der Farbdopplersonografie oder Elastografie weiter gearbeitet.

Dass die Magnet-Resonanz-Tomografie von Deutschland aus einen beeindruckenden Beitrag zur Steigerung der Sensitivität der Mammadiagnostik lieferte, ist mit den Namen Kaiser und Heywang verbunden, die Mitte der 80er-Jahre durch die Verwendung des Kontrastmittels Gd-DTPA den Durchbruch für die MRT der Mamma erzielten. In den 90er-Jahren konnte das Verfahren durch Publikationen vieler Arbeitsgruppen seine Stellung ausbauen und weitgehend definieren. Allerdings steht beispielsweise für den präoperativen Einsatz der MRT der Nachweis des Benefits aus onkologischer Sicht noch aus, da sich in dieser Zeit nicht nur die radiologische Technologie, sondern auch die Behandlung des Mammakarzinoms gewandelt hat.

Der Siegeszug der brusterhaltenden Therapie, der ohne Einbezug von Ultraschall oder MRT eingeleitet worden war, die Wächterlymphknoten-Methode, die adjuvanten (und neoadjuvanten) Therapiekonzepte leisten Beiträge zur Senkung von Mortalität (und teils auch Morbidität), deren Relation zu den vor allem sensitivitätssteigernden Methoden der Radiologie sicherlich in den nächsten Jahren weiter evaluiert werden muss - sicherlich auch weiterhin mithilfe des multidisziplinären Ansatzes einer erfolgreichen deutschen Gesellschaft für Senologie!

\section{Literatur \\ 1 Lanyi M. Diagnostik und Differentialdiagnostik der Mammaverkalkun- gen. Springer; 1986 \\ 2 Frischbier H-J, Hoeffken W, Robra B-P. Mammographie in der Krebs- früherkennung. Ferdinand Enke; 1994}

\section{Bibliografie}

DOI http://dx.doi.org/10.1055/s-0031-1271500

Senologie 2011; 8: 78

(c) Georg Thieme Verlag KG Stuttgart · New York · ISSN 1611-6453

\section{Korrespondenzadresse}

\section{Prof. Dr. med. Markus Müller-Schimpfle}

Ärztlicher Leiter des Instituts für Radiologie

Klinikum Frankfurt Höchst

Gotenstr. 6-8

65929 Frankfurt

mms@skfh.de 